\title{
Purification of filamentous bacteriophage for phage display using size-exclusion chromatography
}

\author{
Maria Yu. Zakharova ${ }^{1}$, Arina V. Kozyr ${ }^{1}$, Anna N. Ignatova ${ }^{1}$, Ilya A. Vinnikov $^{1}$, \\ Igor G. Shemyakin ${ }^{2}$, and Alexander V. Kolesnikov ${ }^{1}$ \\ ${ }^{1}$ Russian Academy of Sciences, Moscow and ${ }^{2}$ State Research Center for Applied \\ Microbiology, Obolensk, Russia
}

BioTechniques 38:194-198 (February 2005)

Phage display has proved itself as a powerful and convenient approach for rapid sampling of billions of proteinaceous structures against a ligand of interest. The most widespread system uses display of proteins and peptides as fusions to coat proteins III or VIII of the M13/fd phage. Efficient screening of phage display library requires high purity of the input phage particles.

Double precipitation by polyethylene glycol (PEG) with an average molecular weight of 8000 Da (PEG8000 ) is a standard technique for purification of filamentous bacteriophages $(1,2)$. Certain contaminants could still remain in the PEG-purified phage preparation. Although efficient removal of contaminating RNases from bacteriophage preparation using treatment by ionic detergent followed by ultracentrifugation in $\mathrm{CsCl}$ gradient was reported (3), this method is costly, time-consuming, and is not applicable if the native state of the phage-displayed protein or peptide is affected by ionic detergents and high salt.

Size-exclusion chromatography (SEC) has been used in preparation of pure eukaryotic viruses $(4,5)$ and bacteriophage $\lambda(6)$. We assumed that SEC can be the method of choice for efficient purification of filamentous phages under mild conditions. In this work we describe a rapid and simple technique for chromatographic purification of filamentous phage particles in Sephacryl ${ }^{\mathrm{TM}} \mathrm{S}-500$ resin (Amersham Biosciences, Piscataway, NJ, USA).

The purity of phage preparations obtained after double PEG precipitation was assayed by electrophoresis in Tris-tricine (7). Phage particles displayed the single-chain variable fragment (scFv) of the MRL-4 antiDNA monoclonal antibody (MAb) (8) fused to the C-terminal portion of the pIII minor phage coat protein ( $\delta \mathrm{pIII})$. Production of the $s c F v-\delta p I I I$ fusion cloned into the pTScDisp phagemid (GenBank ${ }^{\circledR}$ accession no. AY618909) was controlled by the tac promoter. The phagemid was grown in the DH12S Escherichia coli strain (Invitrogen, Carlsbad, CA, USA) induced to express the $\mathrm{scFv}-\delta$ pIII by the addition of 0.1 $\mathrm{mM}$ isopropyl- $\beta$-D-thiogalactopyranoside (IPTG) and rescued by the VCS M13 helper phage using the standard technique (2). The same phagemid was grown and rescued without induction of the $\mathrm{scFv}$ - $\delta$ pIII expression in the presence of $2 \%$ glucose instead of IPTG. Purification of phage particles was done using the standard protocol $(1,2)$, except the first PEG precipitate was resuspended in phosphate-buffered saline (PBS) containing $0.05 \%$ Tween $^{\circledR}$ 20. Purified particles were stored in PBS at $4^{\circ} \mathrm{C}$ until use. After purification, plaque- and colony-forming units per milliliter (pfu/mL and cfu/mL, respectively) were quantitated as described previously (2).

Approximately $5 \times 10^{11}$ pfu of PEGpurified phage particles were mixed 1:1 with the sample loading buffer, boiled for $5 \mathrm{~min}$, electrophoresed, and then the gel was silver-stained. The assay, done in triplicate, showed that phage preparations contained varying amounts of proteinaceous and low-molecular weight impurities. A particularly high level of contaminants was observed at the background of the induced expression of the $\mathrm{scFv}-\delta$ pIII fusion (Figure 1, lane 2).

PEG-purified phages were subjected to the SEC in Sephacryl S-500 resin. The exclusion limit of Sephacryl S-500 $\left(2 \times 10^{7} \mathrm{Da}\right)$ is well suited for separation of filamentous phage particles (approximate molecular weight of $17 \times 10^{7} \mathrm{Da}$ ) from a wide range of potential contaminants. A sample containing $10^{13} \mathrm{pfu}$ in $0.5 \mathrm{~mL}$ PBS was loaded onto the Superdex ${ }^{\mathrm{TM}}$ 200 HR 10/30 column (Amersham Biosciences) packed with $30 \mathrm{~mL}$ of Sephacryl S-500 resin and pre-equilibrated with PBS. Since the typical pfu/cfu ratio was 99:1, only pfus were considered in the calculation of total amount of loaded particles. The optimal amount of the phage preparation that could be loaded to the column without loss of separation efficiency was determined experimentally (data not shown). The column was developed with PBS at $0.4 \mathrm{~mL} / \mathrm{min}$ using a chromatography system equipped by the Model 994 photodiode array detector (Waters, Milford, MA, USA) set at $280 \mathrm{~nm}$.

The 1-mL fractions of the column eluate were collected and assayed for pfu and cfu counts. The elution profile consisted of two peaks (Figure 2). Most of the phage particles were eluted in the first peak ( $4.5 \mathrm{~mL}$ total volume), whereas other fractions contained at least 5 orders of magnitude less phages. The second peak contained various contaminants and PEG. Loss of the phage and phagemid viability after SEC was about $10 \%$ judging by comparison of pfu and cfu counts obtained before and after chromatography. In some experiments SEC-purified phage particles were concentrated using Centricon ${ }^{\circledR}-100$ (Millipore, Billerica, MA, USA) without loss of infectivity. In total, the chromatographic purification took $20 \mathrm{~min}$ to collect the eluted phage, or $1-1.5 \mathrm{~h}$ if the sample concentration was performed.

An aliquot of SEC-purified phage preparation contained $5 \times 10^{11}$ pfu was electrophoretically resolved (7) (Figure 1). Silver staining of the gel revealed single major band corresponding to the pVIII coat protein and faint bands possibly belonging to other phage proteins (Figure 1, lane 1). After SEC, the impurities observed in the PEGpurified phage preparation (Figure 1, lane 2) were efficiently removed.

After demonstrating that SEC 
did not significantly decrease phage viability, we asked if chromatographic purification could reduce panning efficiency by increased shedding of the displayed moiety. The panning efficiency of the SEC-purified phage particles was assayed in three different formats: (i) $\delta$ pIII-based peptide display; (ii) pVIII-based peptide display; and (iii) $\delta$ III-protein display. In all the formats, panning of the phage particles was achieved by using the c-myc peptide tether and the anti-c-myc 9E10 MAb (accession no. CRL-1729; ATCC, Manassas, VA, USA). The construct expressing the c-myc- $\delta$ pIII fusion was prepared by PCR-mediated insertion of the c-myc DNA in the pTDisp vector (GenBank accession no. AY618908) between pelB and $\delta$ gIII sequences. The construct for display of the protein$\delta$ IIII fusion was prepared by inserting the DNA encoded the VIPase catalytic antibody light chain with $\mathrm{N}$-terminal c-myc and His6 tags, pelB leader sequence, and ribosome-binding site (RBS) (9) in pTDisp. The construct encoded the c-myc-pVIII fusion was assembled by PCR and cloned into the pTDisp in place of $\delta \mathrm{gIII}$. Each of the constructs was used to produce c-myc positive $\left(\mathrm{c}-\mathrm{myc}^{+}\right)$phagemid particles.

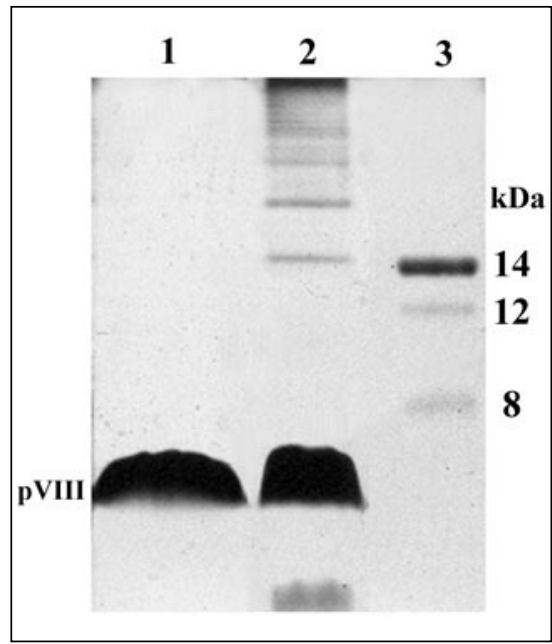

Figure 1. Denaturing Tris-tricine electrophoresis of purified phage particles obtained after rescue of a phagemid displayed the singlechain variable fragment $(\mathrm{scFv})$-p3 fusion. Lane 1 , phage preparation recovered after size-exclusion chromatography (SEC). The major band is the phage coat protein pVIII, molecular weight $6 \mathrm{kDa}$; lane 2, same preparation but purified by double-polyethylene glycol (PEG) precipitation only; lane 3, low molecular weight (LMW) marker (Amersham Biosciences).
The c-myc negative (c-myc $c^{-}$) particles were prepared by rescuing the unmodified pTScDisp with VCS M13. After PEG-purification and quantitation, equal amounts of cfu displaying each of the three fusions were mixed with the c-myc particles at the 1:100 dilution ratios. Half of each mixed pool was purified by SEC and analyzed for cfu/ pfu counts. Judging by electrophoretic analysis, the contaminants found in all the PEG-purified preparations were removed by the SEC (data not shown).

For panning, $10^{9} \mathrm{cfu} / 0.5 \mathrm{~mL}$ from each of the pools were incubated with $100 \mu \mathrm{g}$ of $9 \mathrm{E} 10 \mathrm{MAb}$ for $2 \mathrm{~h}$, than mixed with $100 \mu \mathrm{L}$ of Protein $\mathrm{G}$ Sepharose ${ }^{\circledR}$ (Amersham Biosciences) and incubated for the next $2 \mathrm{~h}$. The unbound particles were removed by repeated washing with PBS, and then bound phages were eluted from the Sepharose with glycine- $\mathrm{HCl}, \mathrm{pH} 2.6$, and used to infect $E$. coli. Infected bacteria were plated, and individual ampicillin-resistant colonies were analyzed by PCR for the presence of the c-myc ${ }^{+}$construct.

In each of the display formats analyzed, similar panning efficiency was observed for PEG/SEC-purified and PEG-purified phagemid preparations. For the c-myc- $\delta$ pIII display, $18 / 100$ c-myc $^{+}$clones were captured from the SEC-purified pool, and 19/100 clones from the PEG-purified one in single round. For a single round of c-myc-pVIII phagemid panning, the respective values were $41 / 100$ and $37 / 100$ of c-myc ${ }^{+}$clones. Two rounds of panning were required to achieve detectable enrichment of VIPasedisplaying phages. After a second round of panning, 11/100 c-myc $\mathrm{c}^{+}$clones were captured from the SEC-purified pool, and 9/100 from the PEG-purified one. Use of SEC did not affect the panning efficiency in the three display formats analyzed. The quantity of phage particles purified at the $30-\mathrm{mL}$ column in a single run $\left(10^{13}\right)$ exceeds the size of the largest reported phage display libraries by 2 orders of magnitude and is sufficient for conducting the panning round. If necessary, the procedure can be scaled up using larger columns.

Modifications of the "classic" phage display, such as the in vitro evolution of enzymatic activity, selection of phages internalizing into eukaryotic cells, and the "substrate phage" technique (see Reference 1 for a review), demand high purity of phage(mid) preparations. Certain enzymes co-precipitating with phages hamper selection of phagedisplayed catalytic activity (3). The E. coli lipopolysaccharide (LPS) and the traces of PEG that remained in the sample could be cytotoxic and thus impeding phage panning utilizing living eukaryotic cells. The contaminants in the phage samples could also interfere with phage enzyme-linked immunosorbent assay (ELISA) employing highly reactive phosphonates (10) and chloromethyl ketones (11) as the

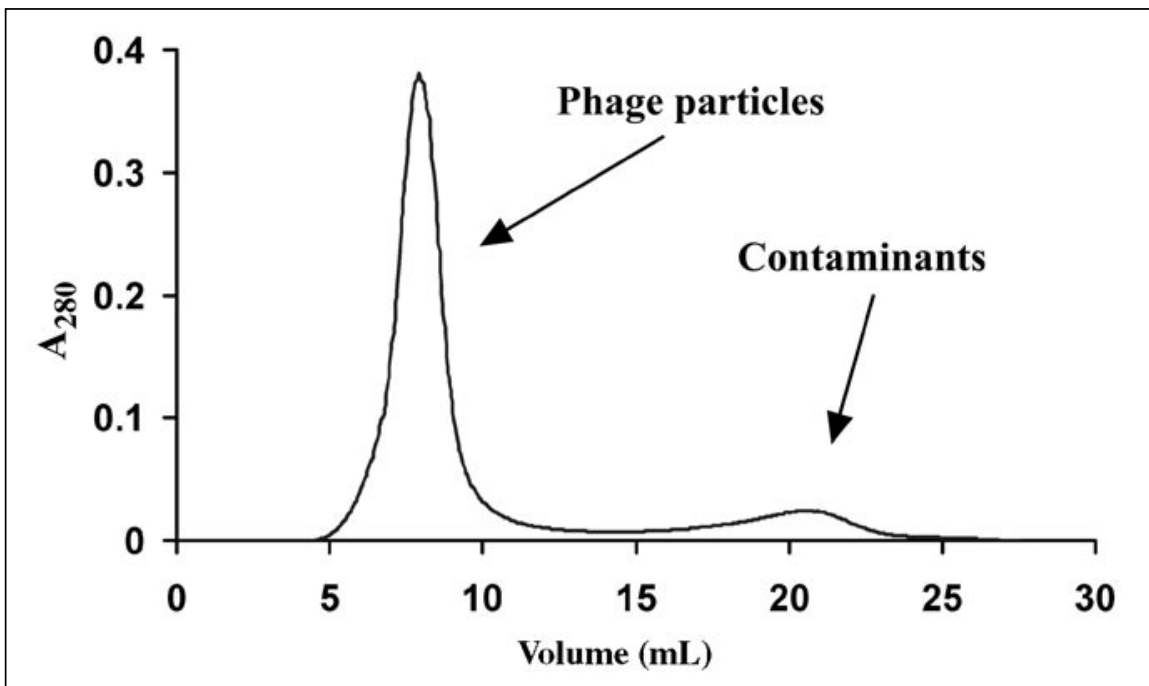

Figure 2. Elution profile of phage preparation subjected to the size-exclusion chromatography (SEC) with Sephacryl S-500. 
probes. The SEC-based method of purification of phage particles is fast and reproducible and yields highly pure phage preparation while preserving the phage infectivity and the integrity of displayed moieties.

In summary, we developed a method by which contaminants observed in the PEG-purified phage preparations can be removed under mild conditions. We hope that the new technique could be useful in various applications of the phage display library approach.

\section{ACKNOWLEDGMENTS}

The work was supported by the Russian Foundation of Basic Research (grant no. 02-04-49411) and by International Science and Technology Center (grant no.1758).

\section{COMPETING INTERESTS STATEMENT}

The authors declare no competing interests.

\section{REFERENCES}

1.Sambrook, J., E.F. Fritsch, and T. Maniatis. 1989. Molecular Cloning: A Laboratory Manual, 2nd ed. CSH Laboratory Press, Cold Spring Harbor, NY.

2.Kay, B.K., J. Winter, and J. McCafferty. 1996. Phage Display of Peptides and Proteins. A Laboratory Manual. Academic Press, San Diego.

3.Korn, K., H.H. Foerster, and U. Hahn. 2000. Phage display of RNase A and an improved method for purification of phages displaying RNases. Biol. Chem. 381:179-181.

4.Loa, C.C., T.L. Lin, C.C. Wu, T.A. Bryan, H.L. Thacker, T. Hooper, and D. Schrader. 2002. Purification of turkey coronavirus by Sephacryl size-exclusion chromatography. J. Virol. Methods 104:187-194.

5.Transfiguracion, J., D.E. Jaalouk, K. Ghani, J. Galipeau, and A. Kamen. 2003. Size-exclusion chromatography purification of high-titer vesicular stomatitis virus G glycoprotein-pseudotyped retrovectors for cell and gene therapy applications. Hum. Gene Ther. 14:1139-1153.

6.Sain, B. and S. Erdei. 1981. Bacteriophage purification by gel chromatography. Anal. Biochem. 110:128-130.

7.Schagger, H. and G. von Jagow. 1987. Tricine-sodium dodecyl sulfate-polyacrylamide gel electrophoresis for separation of proteins in the range from 1 to $100 \mathrm{kDa}$. Anal. Biochem. 166:368-379.

8.Kofler, R., R. Strohal, R.S. Balderas, M.E.
Johnson, D.J. Noonan, M.A. Duchosal, F.J. Dixon, and A.N. Theofilopoulos. 1988. Immunoglobulin kappa light chain variable region gene complex organization and immunoglobulin genes encoding anti-DNA autoantibodies in lupus mice. J. Clin. Invest. 82:852-860.

9.Demin, A.V., A.V. Kolesnikov, M.A. Olfer'ev, P.V. Favorov, K.V. Fegeding, A.G. Gabibov, and N.V. Gnuchev. 1998. Design of constructions for the expression of proteolytic antibody light chain. Genetika 34:1333-1337.

10.Gao, C., C.H. Lin., C.H. Lo, S. Mao, P. Wirsching, R.A. Lerner, and K.D. Janda. 1997. Making chemistry selectable by linking it to infectivity. Proc. Natl. Acad. Sci. USA 94:11777-11782.

11.Atwell, S. and J.A. Wells. 1999. Selection for improved subtiligases by phage display. Proc. Natl. Acad. Sci. USA 96:9497-9502.

Received 29 January 2004; accepted 8 September 2004.

Address correspondence to Alexander $V$. Kolesnikov, Shemyakin and Ovchinnikov Institute of Bioorganic Chemistry, Russian Academy of Sciences, Ul. Miklukho-Maklaya, 16/10, 117997 GSP, Moscow V-437, Russia.e-mail:koleso@ibch.ru 